\title{
An Analogy to Help Understanding Discovery, Insight and Invention in Mathematics
}

\author{
Ruggero Ferro \\ Department of Computer Science, University of Verona, Italy
}

Copyright $\subseteq 2019$ by authors, all rights reserved. Authors agree that this article remains permanently open access under the terms of the Creative Commons Attribution License 4.0 International License

\begin{abstract}
An analogy with how life would be evolving in a town where one is moving in, may help us to understand what could be meant by discovery, insight and invention in mathematics. The relevant key common features of these two environments (life in an another town and mathematics) are: 1) the involved mental abilities to deal with the situation, 2) the realization that anything observed is contingent, 3 ) the discovery of the motivations of what has been done and of their influence up to the present via insight, 4) the need to understand the motivations and the manners of realization of what was done to continue the development, 5) the continuous evolution of needs and requirements that opens new problems that demand insight and invention for their solutions, 6) not every solution meets the goals and requirements with the same short range and long range convenience, thus a preventive evaluation is convenient according to criteria to be established, though a conclusive evaluation can be done only afterward. What observed will justify the support of a dynamic attitude toward mathematics and the refusal of the one claiming that everything can't be but the way it is, according to a priori mental evidence which is unduly assumed.
\end{abstract}

Keywords Mathematics, Discovery, Invention, Insight

\section{Introduction}

Is mathematics discovered or invented? Which is (if any) the role of insight in developing mathematics?

Discovery, insight and invention are not notions formally defined in mathematics, in a way that we can prove some statements about them, but they are notions about the human modalities to approach mathematics. Thus, to capture their meanings, it is convenient to investigate humans behaviors not only with respect to mathematics.

An analogy with how life would be evolving in a town to where one is moving may help us to understand what could be meant by discovery, insight and invention in mathematics.

\section{The Analogy}

Imagine a young person moving to a different town, which is new to him; say because he is hired for a new job there. Going there he discovers many aspects that he didn't know: a new landscape, new buildings and streets, new traffic, new public transportation systems, new cafeterias and restaurants, new department stores, new theaters, new social life; new workplace, new task to perform, new colleagues, and so on. Indeed these are all aspects that were already there; they are contingent (in other places they are different, and even in that place they could have been different and indeed they were different at different times), and he did not know of them before. Thus they are not new in themselves, but new to his knowledge, so that discovering is an epistemic act, it doesn't alter the environment but the knowledge of it.

In his new town, he has to look for a place where to live, and he looks around for apartments for rent. He finds many opportunities, probably too many: a lot of prices, different locations and number of rooms, different layouts, designs, air-conditioning and lighting systems.

There are offers that are too expensive, that he cannot afford, so there is no point in considering them further, even though they may be the best choice considering the value price ratio: even though it is impossible to have all pieces of information (including the future consequences) before taking a decision (thus having to accept some risk in deciding), in case of not affordability this one piece of information could be enough to decide an exclusion.

Still the remaining possibilities are too many and too diversified that, to disentangle himself of this mess of data, he invents a way of organizing all the data to compare them more easily.

In order to get a global picture, he creates, better invents (since are not obtained from nothing), scales and a way of combining them, so to assign a preference grade, according to his taste, to each aspect of each different offer. He tries 
out these scales on some instances to check whether the results obtained match indeed his taste, and, after a couple of tuning of the instrument, it works. Thus, he is able to determine the best offer.

But pretty soon he realizes that by changing the layout of one apartment, he gets an even better solution. He is very proud of himself for the invention of the new layout. Yes, again an invention, being the construction of a new original (not a copy) state of affairs using elements that are there.

When new in town, things around him looked to be as they were, without any reason for their being that way; he was busy enough discovering the situation and probably he had no time for questioning why it was so. He knows very well that his voluntary actions are due to his will, they have a cause, so he assumes that there should also be reasons why that town grew the way it is now, why buildings and streets were constructed as they are, why the prices of commodities are as they are, why the social life is as it is (including locations and layouts of restaurant, theaters, social clubs, cultural agencies, political structures).

The reasonable motivations that could be envisioned are reasonable to us today or were reasonable to those taking the decisions when these were made? Different historians and sociologists may give different accounts of the reasons why the town evolved as it did. Thus we realize that the given answers are hypothetical ones, since we were not there (nor the historians, even though with their studies they can eliminate untenable hypothesis), we are not the persons that took the relevant decisions, and we cannot enter into them to know the intimate motivation that they had. Thus we have to be satisfied with reasonable hypothetical justifications, possibly coming from a deep insight. We are well aware that even the most precise manifestation of our ideas may be honestly misinterpreted, and this is possible since every theory (dealing with an unbound number of elements) has non-isomorphic models. Thus, to understand the original internal intention of somebody, one has to go beyond all the external expressions and manifestations of that intention, and try to figure out what was going on within that person, one has to be empathetic, possibly taking advantage of a deep analysis of one self's attitudes as if he were placed in the other person's situation, mentality, feelings and desires (in the other person's shoes), assuming to be similar to the other person: in one word one needs a deep insight.

Since people's needs and wishes change and can never be completely satisfied, not everything that is there in that town is so adequate to the present situation. Of course poorly constructed artifacts fade away leaving almost no trace, but even what was properly realized responding to now obsolete requirements may demand substantial remodeling. To satisfy the demand new solutions are needed. The demand may present problems unsolvable with the tools at disposal thus requiring inventions that should come from a deep insight of the situation and of the demands in order to respond to the requests at best: indeed insight doesn't concerns only the scrutiny into other persons but also into the activities and products of groups of persons to capture motivations and goals.

\section{Mathematics}

Mathematics' development has a very similar outline.

At a first approach with mathematics, one meets problems, notions, techniques, and results that are there: they were already developed and one has to learn them discovering their features and usefulness. This process will take some time because the material available is very rich and complex, after millennia of developments. What is learned is new to the learner in the sense that he had no previous knowledge of it. [1]

It could appear as if every aspect was already considered and fully developed: indeed a careful search in the literature may clarify most doubts and problems that could arise. Furthermore one is impressed by amount and depth of already acquired results, and starts wandering how it was possible to reach such accomplishments. Looking at the history of the subject, [2] one comes to appreciate the hard work that was put in its development, the different approaches to the problems at hand, the emergence of reasonable attitudes and results with respect to prospected goals. One also learns of open problems (i.e. problems for which at the moment no one knows a solution), even some that can be elementarily stated, and is guided to invent solutions to some of them. Presenting a proof that the conclusion holds in any possible situation in which the hypotheses of the problem are respected is a certification that the problem is solved. Indeed, rather than the same notions, the important tools used are the characteristics of those notions captured by the hypotheses. Whence the attitude to minimize the effort in understanding the notions to favor the relying on their explicitly declared characteristics.

However, after having reached (or studied) a result relying only on some hypothesis, one may wonder about its relevance. To appreciate the latter, one needs to have an idea of the intended meaning of the entire subject, beyond its explicit statements. Indeed, most of the interesting mathematical objects cannot be univocally determined by their explicitly declared properties in a given language, due to the existence of non-isomorphic models satisfying all of those properties, no matter how rich the language is. [3]

We can agree that the scholar in mathematics discovers notions already elaborated by others, but how could the first person that introduced a notion have had a grasp of it? The relevant mathematical notions (numbers, figures, relations, functions, spaces, etc.) are not concrete, so they cannot be perceived by the physical senses. To be discovered they have to be already there, but where? Indeed Kronecker [4] claimed that the natural numbers are there, and, to avoid specifying where, he added that they 
were given by God. I dare say that Kronecker had no insight in the notion of natural number; just he didn't care to analyze it, satisfied with the ability of using the numbers. Peano [5] was more careful and realized that fundamental properties of the notion of natural numbers system can be enucleated to become (he thought) its so powerful axioms to univocally characterize (up to isomorphisms) the same notion. Unfortunately, he didn't notice that he was not considering all the possible models but just the full ones for only any two full second order structures that satisfy the Peano's axioms can be proved to be isomorphic. [6]

A more careful analysis and insight into the notion of natural number shows that it is constructed through mental operation starting from concrete experience: hence it should be considered an invention.

Indeed, way before using numbers, humans had the experience of comparing two sets of concrete elements asserting that they have the same quantity of elements if there is a bijective function (briefly a bijection) between the two.

There are cases in which pairing the elements of one set with those of the other to build a bijection is trivial, but it is not always so. When it is difficult, an invented good strategy to construct a bijection prescribes to consider an element to be paired at a time in each one of both sets and to continue this way with further elements in both sets until all the elements of one set are considered.

Since the composition of bijections is a bijection the strategy can be improved and the quantity of elements of a set can be compared with well manageable standards of quantities, for instance the repetitions of the mental operation of considering a further element while considering the elements of a set one by one (of course one has to separately remember each one of these acts, and if the memory is not powerful enough it can be aided with marks). These standards for quantities may be considered as the natural numbers. [7]

Notice that this introduction of the natural numbers is not an explicit definition but a description of a process that can be performed within each individual (maybe in slightly different ways according to the different individual past experiences), and each one will grasp his own notion of natural number, and agree that the axioms that pretend to characterize this notion are appropriate for his notion. This is not so surprising since the individual construction began from the same concrete problem of comparing quantities of elements adopting a reasonable strategy that can be described with sufficient precision.

One could remark that we are considering sequences of actions as elements, but there should be no wonder in doing this, since we are well accustomed and able to do so, even in non-mathematical settings. Walking is a common concrete experience from which we obtain the notion of a walk. We use this notion as an element in expressing statements as "a long walk" or "an easy walk", without pretending a walk to be a concrete object. I claim that the same acceptable type of mental construction from experience is performed in acquiring the notion of natural number.

What I tried to portray is only a first approach and insight into the notion of natural numbers, since many questions remain unanswered so far. For instance, how large is the amount of the natural numbers? Humans have only experience of processes that end, but it is not convenient for the proposed goal to assume that there is a largest natural number. Thus the idea of an unending (infinite) quantity of natural numbers was invented, with all the attached difficulties that come from the non-controllability of the situations thus accepted.

In order to cooperate humans need to communicate, and they invented and negotiated words and an evolving language to indicate the interesting elements and the relations among them. In the case of mathematics, languages and notations were invented so closely linked to the indicated elements and relations that it was possible to determine transformations on the sequences of symbols that correspond to operations on the indicated notions: calculi were invented. Calculi are very important because they provide a precise control on operations among notions the outcome of which could be hardly detected and foreseen.

Indeed, humans are unable to cope with complication, i.e. they are unable to master all the pieces of information coming from the environment (from reality) which are too many and disordered. So they consider only some of the pieces of information (they abstract the ones considered relevant) constructing a model of reality hopefully close enough to reality, but the model necessarily differs from reality. To mimic reality at best, models accept large quantities of data, but they organize them in order to make models manageable, thus introducing complexity. Humans have a hard time dominating complexity too, but good strategies can be devised to control and manage such models and calculi are very good examples of such strategies, since they are adequately well organized even though they are complex. [8]

Clearly this is not the moment to dwell on the details of the modeling processes outlined. I presented some aspects of the acquisition of the notion of natural number as a paradigmatic example of what happens also in the case of other mathematical notions, and I claim that the one portrayed is a good picture of the way most mathematical notions are constructed. Among these, let me just mention the case of the intuitive experiential notion of continuity (i.e. without interruptions) that has been developed in different incompatible but equally acceptable manners (either introducing or banning the infinitesimals), each manner being more convenient with respect to different intended specific goals.

Up to now, I mainly considered discoveries, insight and inventions of mathematical notions, but in the development of mathematics we meet also arguments, proofs, theorems 
and theories about those notions. These are established in a peculiar way as to give mathematics its flavor of solidity and certainty to become a paradigm of scientific rigor for all other sciences: once obtained a proof of a theorem it holds forever beyond any doubt. This goal is possible since a proof is finally formulated in a language and it can be completely checked and controlled.

Nevertheless it could be so complicated, involved and extended to make it very hard to follow it. Hence here again anyone who is studying it has to discover the way it proceed. A help can come from the insight in the line of thought followed by the proof's author. In order to limit their length and increase readability, most advanced proofs are based on other proofs, results and entire theories are presupposed to justify several steps in the same proof. This type of presentation certainly makes it possible to follow more easily the flow of the proof, but limits the readership to those who already know all that is assumed.

On the other hand, the author of a proof should invent the steps to perform to reach the goal, guided from the insight he has of the subject at hand. The job of inventing new proofs, which is the main task of working mathematicians, is not trivial at all. Indeed no machine will ever be able to perform it since the set of all theorems (i.e. sentences deducible from the assumptions) is recursively enumerable but not recursive. Thus once a pretended proof of a statement is presented it is possible to effectively check its correctness, but to invent proof requires ingenuity. Furthermore, not all statements are equally interesting and helpful to give a more workable and productive picture of a subject matter, so a deep insight in their meaning is needed to select the relevant statements: it is not without reasons that proved statements are classified as exercises, lemmas, theorems, corollaries, fundamental theorems.

Thus even in the typical mathematical activity of proving relevant results discovery, invention and insight play crucial roles, even though it is hard to see a counterpart to this activity in the analogy proposed.

\section{Common Aspects}

I chose to present the analogy between the two very different states of affairs to point out that in both cases we have discoveries, inventions and insights. The possibility of looking at these actions from different points of view enriches, through more experiences, the understanding of them.

The mental abilities required to perform these actions are the same in both cases. They include:

a) memory (to store, retrieve and compare the data received from the external and internal senses and from previous mental elaborations),

b) abstraction (to simplify the situation considered keeping only the relevant data), c) generalization (to claim that what was seen to always happen under certain conditions, will happen under the same conditions even when it is not experimented),

d) idealization by addition (to put together aspects captured in different disjoint situations to characterize a new notion),

e) idealization by negation (to add the negation of a known aspect to construct a new notion),

f) introspection (to be aware of the relevant role of internal senses both in constructing one's own notions and in gaining sensibility about other people's points of view). [9]

Probably, in the case of mathematics, these abilities are sharpened due to their intensive use.

The analysis so far developed shows that, rather than the tools used, what differentiates mathematics from other fields is its scope, which consists in organizing multiplicities to manage them.

The two scenarios that were considered allow us to notice some general features.

1) It is very reasonable to suppose that anything that was build responded to some need, requirement, convenience or development.

2) The previous achievements have some influence and bearing on what is done afterwards;

3) An understanding of the motivations of what was done and of the manners in which it was realized is very convenient to positively continue the development even in opposite directions.

4) The needs and requirements are continuously evolving and newly invented artifacts or improvements would be relevant tools to cope with them.

5) Not every invented development satisfies the requests with the same short range and long range convenience, thus a preventive evaluation would be welcome to minimize possible drawbacks even though a conclusive evaluation is possible only afterwards.

\section{Conclusions}

All together, I am proposing and trying to make emerge an attitude towards mathematics that is rather different from the popular one that consider mathematics as totally certain, without novelties to propose. On the contrary mathematics should to be considered contingent, as any other human knowledge, since humans are unable to determine universal absolute notions due to their limits to cope with complication. Thus even in mathematics one has to take risks in proposing notions that are initially based on assumptions, notions that could turn out to be of no interest and usefulness, even contradictory. Even though proofs and theorems are there to stay, as events in the past that cannot be changed, they aren't but the link from 
assumptions to conclusions and they depend on their hypothesis. These can be more or less adequate to model and analyze notions and situations which are not absolute. Indeed one has to be ready to change the hypothesis as needed to obtain notions more adequate to the continuous deepening and expanding of the subject matter. The proposed attitude towards mathematics should not obscure the other aspects of mathematics which account for most of the work done in the subject by mathematicians: once axioms and definitions are agreed upon, the derivation of statements that are relevant theorems is an invention task of great value since their totality is not producible nor evaluable by any ideal machine, even though their correctness is ideal-machine controllable.

\section{REFERENCES}

[1] R. Courant, H. Robbins: What is Mathematics? Oxford University Press, London, 1941

[2] M. Kline: Mathematical thought from ancient to modern times, Oxford University Press, New York, 1972

[3] A.J. Cuneo, R. Ferro: Logica matematica elementare classica: potenzialità e limiti del linguaggio, ARACNE, Roma, 2019, ISBN 978-88- 255-1761-3

[4] L. Kronecker: Werke, AMS Chelsea Publishing, 1968, ISBN 978-0-8284-0224-8

[5] G. Peano: Aritmetica generale e algebra elementare, Paravia, Torino, 1902

[6] A.Robinson: Nonstandard analysis, North Holland, Amsterdam, 1974, ISBN 0720422000

[7] A.J. Cuneo, R. Ferro: Matematica elementare: unapproccio critico, forthcoming

[8] R: Ferro: Reality, knowledge of reality, representation od the knowledge of reality, in E. Agazzi: Representation and explanation in the Sciences, Franco Angeli, Milano 2013, pp. 94-106. ISBN: 9788820420130

[9] A.J. Cuneo, R. Ferro: "From the Classroom: Toward A New Philosophy of Mathematics", in B. Gold, C. Behrens, R. Simon eds: "Using the Philosophy of Mathematics in Teaching Undergraduate Mathematics", MAA Notes 86, Mathematical Association of America, 2017, pp. 257-269, ISBN 978-0-88385-196-8 